Technology and rule from Pierre Bourdieu's social theory

\title{
Tecnología y dominación desde la teoría social de Pierre Bourdieu
}

\author{
José Manuel Iglesias Granda \\ Investigador FPU en el Instituto de Filosofía del CSIC \\ josemanuel.i.granda@csic.es
}

DOI: https://doi.org/10.15366/bp2021.27.014

Bajo Palabra. II Época. No 27. Pgs: 273-290

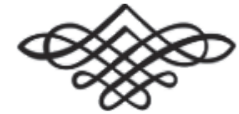


Recibido: 30/06/2020

Aprobado: 07/05/2021

\section{Resumen}

El objeto de este artículo radica en rescatar el planteamiento teórico de Pierre Bourdieu de cara al estudio de las posibles formas de dominación en las que la tecnología puede jugar un papel importante. En ese sentido, a partir de los conceptos bourdianos de "habitus", "tiempo" y "capital" se profundiza en las siguientes cuestiones: 1) la relación entre tecnología y precarización; 2) la posibilidad de un horizonte disruptivo utópico; 3) la relación entre capital económico y las diversas formas de capital tecnológico.

Palabras clave: Bourdieu, capital, dominación, habitus, tecnología, tiempo.

\section{Abstract}

This paper intends to apply the Bourdieu's sociological theory to the problem of technological rule. In that sense, the concepts of "habitus", "time" and "capital" are taken into consideration so as to study three main questions: 1) the conection between technology and deprivation; 2) the possibility of an utopian disruptive horizon; 3) the relation among economical capital and the several ways of technological one.

Keywords: Bourdieu, capital, domination, habitus, technology, time, rule. 


\section{Introducción ${ }^{1}$}

El BINOMIO TÉCNICA-DOMINACIÓN es quizá una de las problemáticas filosóficas más fecundas de los últimos dos siglos. Por tanto, no puede decirse que su actualidad hoy sea algo sorprendente. Ahora bien, sí que es cierto que la magnitud del desarrollo tecnológico que presenciamos y que nos sobrevendrá confiere gran importancia a esta reflexión en el presente.

Yendo más allá de definir un posicionamiento dentro del debate entre los partidarios y los críticos con la tecnología, y también de sus típicas argumentaciones y discusiones relativas a la sustitución hombre-máquina, la renta básica o los peligros de internet..., el aim motive de este artículo radica en rescatar el planteamiento teórico de Pierre Bourdieu de cara al estudio de las posibles formas de dominación en las que la tecnología puede jugar un papel importante. Se considera valiosa su aportación ya que permite rastrear planos del medio social y del ámbito individual que de otra manera pudieran quedar inexplorados. Con ello, no se pretende realizar un estudio sistemático acerca de la cuestión, sino simplemente aplicar ese utillaje conceptual de cara a algunas de las principales líneas actuales de debate.

Para ello, se ofrecerá, en primer lugar, una aproximación sintética a la obra de Bourdieu - profundizando en tres conceptos considerados de especial utilidad para la cuestión-y posteriormente se tomarán esos conceptos para ahondar en algunos de los aspectos más problemáticos de los debates actuales. Concretamente, se tomarán en especial consideración: 1) la relación entre tecnología y precarización; 2) la posibilidad de un horizonte disruptivo; 3) la relación entre capital económico y las diversas formas de capital tecnológico.

\section{En el horizonte de la Cuarta Revolución Industrial}

EXISTE Un CONSENSO GENERALIZADO en torno al hecho de que el avance de las tecnologías y la digitalización generará cambios sociales y estructurales importantes en un futuro próximo. Parece más que probable que cuestiones como la del trabajo,

\footnotetext{
${ }^{1}$ Quisiera transmitir mi agradecimiento al Prof. Eduardo Zazo (coordinador del Máster en Filosofía de la Historia) cuya atención y dedicación han sido ejemplares y, por supuesto, fundamentales para el desarrollo de esta investigación.
} 
la administración, el suministro de energías o las comunicaciones experimentarán transformaciones de gran relevancia; en ese sentido, la denominada "IV Revolución Industrial" ha sido objeto de la preocupación de gran número de intelectuales, políticos y economistas, existiendo ya una rica producción a su respecto. De entre los innumerables intelectuales que vienen tomando parte de este debate, se barajan aquí las ideas de tres de ellos -de especial interés dado el diferente posicionamiento ideológico de los mismos-: Jeremy Rifkin, Evgeny Morozov y Susan George. A partir de ellas, se pueden definir tres líneas principales de reflexión en torno a las implicaciones sociales de las nuevas tecnologías.

\subsection{Relación entre tecnología y dominación}

LA PRIMERA DE elLAS es la relación entre tecnología y precarización. Como hemos apuntado, los autores que barajamos coinciden en señalar el papel fundamental que las nuevas tecnologías tendrán en la conformación de la sociedad. Ahora bien, también es verdad que difieren a hora de apuntar a las repercusiones. Mientras que algunos son muy optimistas otros ven la situación desde una postura mucho más crítica. Jeremy Rifkin aboga por la idea de que las nuevas tecnologías terminarán por colapsar el núcleo del sistema capitalista (concepto de productividad) dando lugar a una nueva forma de ser en el mundo basada en los conceptos de procomún, "horizontalización" de la propiedad y energía renovable -Green New Deal- ${ }^{2}$. No obstante, Rifkin construye su bien intencionada teoría en base a tesis filosófico-históricas que sin lugar a dudas pueden ser cuestionadas; quizá la más cuestionable sea su defensa de una hipotética evolución progresiva de la empatía en la historia humana. ¿No corren estos planteamientos el riesgo de quedarse flotando en una atmósfera "buenista" sin profundizar de veras en el dinamismo de la realidad?

A juicio de Morozov ${ }^{3}$, planteamientos como los de Rifkin son especialmente peligrosos en el sentido de que, al considerar que la humanidad avanza por sí misma hacia metas casi utópicas, promueven el inmovilizo y, por consiguiente, impiden que se tome el control sobre los cambios tecnológicos. El pensador bielorruso señala, por el contrario, que la influencia de la tecnología puede jugar un papel fundamental en la precarización general de las formas de vida y en la desaparición de los estados de bienestar. ¿ Se ha parado Rifkin a pensar en la fuerza que las empresas tecnológicas tienen no solo al controlar el inmenso volumen de datos que se producen

\footnotetext{
2 Rifkin, Jeremy, La sociedad de coste marginal 0, Barcelona: Paidós, 2018.

${ }_{3}$ Morozov, Evgeny, Capitalismo big tech. ¿Wellfare o neofaudalismo digital?, Barcelona: Enclave de libros, 2018.
} 
sino también al ser ellas mismas el único cauce por medio del cual se desenvuelve cada vez más intensamente la vida de las personas? A este mismo respecto, vale la pena traer también a colación al sociólogo italiano Sergio Bologna; en su libro The rise of the european self-employed workforce $e^{4}$, hace especial hincapié en la figura de los trabajadores autónomos y de los actuales freelancers. Estos últimos pueden considerarse el icono de las nuevas formas de empleo y, según Bologna y a pesar de las observaciones de Rifkin, sus condiciones laborales y de vida dejan mucho que desear, suponiendo una clara regresión con respecto al pasado.

Con todo, parece más que evidente que las nuevas tecnologías no están exentas de formas de dominación más novedosas e ingeniosas. En ese sentido, se vuelve especialmente importante estar alerta para que las promesas se tornen en desengaños. Como muy bien señala Morozov, en un momento en que de forma cada vez más intensa la vida social y laboral se desarrolla por medio de cauces tecnológicos, no hay que perder de vista a los que controlan esos cauces y a los intereses que puedan tener detrás. Y es que, desgraciadamente, muchas veces no coincidirán con los de la ciudadanía.

\subsection{La posibilidad de un horizonte disruptivo}

LA SIgUIENTE PROBLEMÁTiCA a tratar es la posibilidad de que la inminente revolución tecnológica pueda abrir la oportunidad a un horizonte disruptivo en el sentido de dejar atrás el capitalismo neoliberal. Aquí vuelven a tener un papel preponderante las teorías de Rifkin quien, como se ha señalado, aboga por el colapso del sistema capitalista y por su progresiva fusión con un sistema comunitario de corte "glocal ${ }^{\text {" }}$ sustentado en las nuevas tecnologías y principalmente en el internet de las cosas y de la energía. Según su modo de ver, la combinación entre la producción con coste marginal 0 y la eclosión de la Internet de la Cosas afectaría de lleno al corazón del capitalismo abriendo la posibilidad del surgimiento de nuevas formas de vida en las que la acumulación de capital, el consumo y la propiedad privada vayan perdiendo cada vez más importancia ${ }^{6}$.

La visión de Rifkin augura, por tanto, una auténtica revolución antropológica... y es que tanto el individualismo como la propiedad privada vienen siendo rasgos fundamentales de la personalidad moderna. Para él, este cambio en la matriz comunicación-energía trastocaría de pleno el concepto de productividad y con él la viabi-

\footnotetext{
${ }^{4}$ Bologna, Sergio, The rise of the european self-employed workforce, USA: Mimesis, 2018.

5 Glocal: término acuñado por Rifkin que conjuga los de "global" y "local".

${ }^{6}$ Rifkin, Jeremy, La sociedad de coste marginal 0, Barcelona: Paidós, 2014.
} 
lidad del capitalismo. La coyunda entre internet y el abaratamiento de las energías renovables generaría nuevos marcos de vida, donde ya no solo no se dependería del capital para la obtención de energía, sino que también el mercado se vería cada vez más mermado en fuerza debido a la compartición gratuita de recursos vía internet y a la producción casera a bajo coste gracias a las impresoras $3 \mathrm{D}$ y otros utensilios.

El crack del capitalismo debido al coste marginal 0 colocaría, por tanto, al hombre en una situación de total auto-transformación. Y lo que es más importante, ante la tesitura de construir definitivamente una sociedad más sostenible y justa. No obstante, como bien señalan los críticos a este tipo de visiones, Rifkin es excesivamente optimista tanto en lo relativo al crack del sistema como en lo relativo a las posibilidades reales del hombre para auto-transformarse en una situación de disrupción --sea del tipo que sea-. Susan George es especialmente clara a la hora de señalar como todos los posibles y cambios y disrupciones son valorados y utilizados por aquellos que ahora ostentan el poder económico y concentran el capital ${ }^{7}$. Con todo, el planteamiento de Rifkin sigue siendo bastante ingenuo al considerar que efectivamente el hombre será capaz de orientar utópicamente los cambios futuros. Ya no solo porque quienes realmente tendrán la oportunidad de hacerlo no contemplan en absoluto esa línea, sino también porque el hombre mismo no puede transformarse a sí mismo de la noche a la mañana y mucho menos dejar de lado cuestiones tan nucleares como aquellas que Rifkin señala. La transformación humana es mucho más progresiva que disruptiva... al menos aquella transformación que conduce a horizontes más esperanzadores. Ya que éstos suelen requerir de madurez y crecimiento personal... cosas que no se obtienen de la noche a la mañana por muy fuertes que sean los cambios.

\subsection{La relación entre capital económico y las diversas formas de capital tecnológico}

EN ESTRECHA RELACIÓN CON LO ANTERIOR se encuentra la cuestión de la horizontalización de los medios de producción y de la concentración del capital que señala Rifkin. De hecho, el núcleo de su teoría radica precisamente en que la mentada coyunda entre nuevas tecnologías y energías renovables dará lugar a una nueva forma de organización del capital totalmente diferente a la propia del capitalismo. A su juicio, el abaratamiento de la producción de energías verdes abrirá la posibilidad del abastecimiento energético a nivel local y comunitario, no siendo necesaria la concentración de grandes dosis de capital para producir energía como lo es

7 George, Susan, Los usurpadores, Barcelona: Deusto, 2014. 
actualmente. Esto, sumado a los avances de las nuevas tecnologías que permitirían la mecanización y el control inteligente de la producción y el consumo sería precisamente lo que permitiría la nueva organización "glocal" rifkiniana.

De nuevo, y al igual que en los dos epígrafes anteriores, las críticas a este planteamiento son abundantes. Cabe de nuevo traer a colación a Susan George quien en sus Informe(s) Lugano I y II y en su libro Los usurpadores ${ }^{8}$ hace una aguda crítica a las formas en que las nuevas multinacionales -especialmente energéticas- están valiéndose de todos avances para caminar en la dirección de una mayor influencia y control en los ciudadanos y en su consumo. También Morozov apunta audazmente a la monopolización de estos avances por parte de las empresas tecnológicas y, por lo tanto, a la imposibilidad de que realmente sean los ciudadanos los que capitaneen un cambio en la producción energética y en el reparto del capital.

De todo esto, puede concluirse que los supuestos "avances" sociales que la IV RI pueda deparar son realmente cuestionables. Ahora bien, y a fin de despejar toda duda y profundizar más en la cuestión, consideramos fundamental plantear a nivel teórico estos problemas. A ese respecto, consideramos que la teoría social de Pierre Bourdieu puede resultar de gran utilidad. A demostrarlo dedicaremos lo que resta de este artículo.

\section{Somera aproximación a la teoría social de Bourdieu. Los conceptos de "habitus","tiempo" y "capital".}

Pierre Bourdieu ha sido un autor especialmente prolífico. Grosso modo, su obra podría comprenderse como un intento de superación del dualismo objetivismo-subjetivismo y del pensamiento sustancialista — representado especialmente por el marxismo- ${ }^{9}$. Dado que explicar en detalle las coordenadas de su obra excedería nuestro trabajo, nos ocuparemos de los tres conceptos clave que, a nuestro juicio, pueden iluminar más efectivamente la problemática que nos ocupa.

\subsection{El "Habitus"}

Metafóricamente, podríamos relacionar el "habitus" con la ósmosis, pero... ¿Cómo se da ésta? Pues bien, según las observaciones de Bourdieu, se adquiere un

\footnotetext{
${ }_{8}$ Véase principalmente: George, Susan, Informe Lugano II, Barcelona, Deusto: 2013 y George, Susan, Los usurpadores, Bercelona, Deusto 2014.

9 Bourdieu, Pierre, "Espacio social y poder simbólico”, en Bourdieu, Pierre, Cosas dichas, Barcelona: Gedisa, 1988, pp. 127-142.
} 
conocimiento por el cuerpo que hace posible la comprensión práctica del mundo. De alguna forma, lo que se pretende transmitir es que el mundo se encarna en el individuo a través de la "incorporación de las estructuras sociales como estructuras de disposición de posibilidades objetivas en forma de expectativas y anticipaciones", de manera que "se adquiere un conocimiento y un dominio práctico del espacio circundante" de carácter inconsciente ${ }^{10}$. Imaginemos a un buen jugador de futbol que ha interiorizado las posiciones del campo y las diferentes tácticas de control y recepción del balón; de la misma manera, el "habitus" es un "saber" que, antes que la conciencia, da lugar a una intencionalidad sin intención que tiene un dominio práctico del mundo que hace posible adelantar el porvenir sin la necesidad de presentarlo como tal. Este saber se aprende, como dijimos, por medio del cuerpo; a través de -diciéndolo con Bourdieu "11 "la confrontación constante a las transacciones afectivas con el entorno social" ${ }^{12}$. Todo esto, nos lleva a constatar que lo social existe por partida doble en forma de instituciones y "habitus". Así, el cuerpo está en el mundo social, pero el mundo social está también en el cuerpo, generando en él un sentido práctico.

\subsection{El "tiempo"}

El INDIVIDUO LLEVA, pues, incorporadas las lógicas del mundo social que ha ido adquiriendo progresivamente en sus experiencias vitales, y esa lógica define -inconscientemente- sus posicionamientos en el presente y en el futuro. A partir de esto, se observa ya la íntima relación que tiempo y praxis tienen en el pensamiento de nuestro autor: el tiempo es el resultado de una elaboración que atañe a las disposiciones y a la práctica y no a la conciencia pensante. Diciéndolo con sus propias palabras: "la práctica no está en el tiempo sino que hace el tiempo"13; ahora bien, esto nos lleva a romper con las concepciones tradicionales que entienden el tiempo como una realidad preestablecida o como una especie de marco newtoniano o a priori kantiano. El tiempo no es el marco vacío en el que se construye un proyecto vital racional, sino que se parece más bien a una constante relación con el presente percibido a través de movimientos de "interés-desinterés, actualización-inactualización, presentificación-despresentificación”.

\footnotetext{
${ }^{10}$ Bourdieu, Pierre, Meditaciones Pascalianas, Barcelona: Anagrama, 1999, p. 173.

11 Ibid. p. 186.

12 Mauss había demostrado ya cómo a través de la inculcación de las técnicas corporales cuerpo a cuerpo se transmite la cosmología moral de una sociedad y a través de ella se inculcan las relaciones de dominación arbitrarias. Véase: Mauss, M., “Concepto de técnica corporal”, en Sociología y Antropología, Madrid: Tecnos, 1979.

13 Bourdieu, Pierre, Meditaciones Pascalianas, op. cit. p. 275.
} 
Por tanto, el inmenso abanico de opciones que constantemente podrían presentarse a la acción es resuelto inconscientemente según las normas sociales incorporadas; y únicamente en las situaciones de crisis se rompe esa suerte de continuidad sociedad-habitus-acción. Tiempo y juego van íntimamente de la mano, de manera que el tiempo se hace a medida que el individuo va realizando sus aspiraciones y expectativas a partir de las opciones ofrecidas por el campo social. El individuo que está inmerso en el juego espera de él la satisfacción de las expectativas que el mismo juego ha grabado en su interior... el tiempo para Bourdieu se entretejería a partir de esa especie de comunión ontológica existente entre persona y campo social. Ahora bien, ¿qué ocurre en esas situaciones en las que se quiebra la coincidencia automática entre illusio y lusiones? En ese momento, según nuestro sociólogo, se produce una negación del presente $-\mathrm{y}$ por ende del habitus incorporado- $\mathrm{y}$ se abre el horizonte de su superación. Ahora bien, la correspondencia aspiración-posibilidad es altamente funcional y difícilmente se produce la crisis o ruptura; aquí es donde cabe comprender el papel del poder como legitimador del capital simbólico, y las diversas formas de dominación que de ello se derivan.

\subsection{El "capital"}

Partiendo de la anteriormente eXplicada REALIDAd práctica del tiempo, para Bourdieu el mundo social es historia acumulada ${ }^{14}$. Y lo es, precisamente, en el sentido de que las continuas acciones o trabajos en los que se va haciendo el tiempo cristalizan en forma de capital. De esta manera, el capital aparece como una fuerza inherente a las estructuras objetivas y subjetivas; y adquiere un importante papel regulador dentro del juego social.

Ahora bien, para Pierre Bourdieu el capital se dice de muchas maneras. El francés discrepa profundamente con el marxismo en su reducción del capital al mero capital económico. Según nuestro sociólogo, "el concepto científico-económico de capital -invención del capitalismo a su parecer- reduce el universo de las relaciones sociales de intercambio al simple intercambio de mercancías" ${ }^{15}$. Bourdieu encuentra ahí una simplificación distorsionadora y llama a hilar más fino dado el riesgo de dejar fuera a un gran número de prácticas sociales que no son reconocidas como económicas en la realidad -a no ser por un gran esfuerzo de enfemización-. Es decir, nuestro sociólogo -que no refuta la primacía de lo eco-

\footnotetext{
${ }_{14}$ Bourdieu Pierre, Poder, derecho y clases sociales, Bilbao: Desclée Brouwer, 2001, p. 131.

15 Ibid. p. 133.
} 
nómico- señala que existe en la sociedad una clara tendencia a la negación de ésta, la cual se manifiesta en el surgimiento de diferentes campos sociales y tipos de capital que revisten un carácter económico pero que no son reconocidos ni reconocibles como tal ${ }^{16}$.

Por consiguiente, el estudio bourdiano del capital tiene una doble dirección: la primera va en la línea de distinguir los diferentes tipos de capital reconocido (capital económico, capital cultural y capital social) y la segunda en la de prestar especial atención en la forma en que esos capitales pueden transmutarse -principalmente en capital económico- . Dejar de lado esto, supondría desechar múltiples universos y juegos de poder en los que los diferentes capitales se entrelazan casi sin distinguirse dando lugar a amplias gamas de tipos de dominación.

\section{Actualidad de los conceptos de Bourdieu.}

LA GLOBALIZACIÓN Y LA PÉRDIDA DE PODER efectivo por parte de los estados a favor de las diversas formas de capital -sea la banca o las empresas trasnacionales- -parece restar fuerza a las teorías de Bourdieu dado el papel central que otorga al Estado ${ }^{17}$. A pesar de esto, sus trabajos siguen teniendo gran actualidad dado que sus principales conceptos pueden ser aplicados satisfactoriamente de cara al análisis del presente; de hecho, en este apartado se presentan tres consecuencias que se siguen de la aplicación de los conceptos de Bourdieu a la problemática de la hipotética Revolución Tecnológica.

\subsection{Habitus, capital simbólico y nuevas formas de dominación}

RECORDANDO LAS EXPLICACIONES acerca de la noción de "habitus", éste se generaba a partir de la progresiva encarnación en el individuo de las características del medio social y estaba en íntima relación con el poder y la instrucción a partir del uso del mismo. De cara al momento presente -en el que algunos ya hablan de Internet como una nueva ágora-, conviene analizar y prestar especial atención a las instancias que pueden actuar como legitimadoras de capital simbólico y a la forma en que, obviamente, influirán en la conformación de los "habitus" de la población. Si la pregunta de antaño con respecto a los Estados radicaba en quién/quiénes habían

\footnotetext{
${ }_{16}$ Bourdieu, Pierre, Poder derecho y clases sociales, op. cit. p. 134.

${ }^{17}$ Fernández, José Manuel, "Capital simbólico, dominación y legitimidad. Las raíces weberianas de la sociología de Pierre Bourdieu”, en Papers Revista de Sociología, vol. 98, n 1, 2013, pp. 51 y ss.
} 
logrado universalizar sus intereses por medio del Estado, hoy día cabe preguntarse si otros intereses concretos puedan estar universalizándose a través de otras instancias. Y también si los Estados mantienen la fuerza suficiente como para vigilar esa cuestión.

Max Weber hacía un diagnóstico pesimista de la modernidad cuando hablaba de la jaula de hierro en que había quedado introducido el individuo al incorporar definitivamente en su ser el carácter ascético del trabajo ${ }^{18}$. Los planteamientos de Bourdieu nos alejan de tomar por definitiva ninguna situación, y por eso tienen potencial para abrir nuevos horizontes; si las reflexiones de Weber conducían a un futuro pesimista, las de Bourdieu lo hacen a un futuro indeterminado, con la responsabilidad intelectual que eso supone. $\mathrm{Y}$ es que, obviamente, el momento presente y el futuro que se avecina prometen abundantes cambios -en forma de digitalización, internet de las cosas, internet de la energía - y éstos pueden ser positivos o negativos.

De cara al análisis de esta cuestión, resulta de especial interés recurrir a la relación habitus-capital simbólico que Bourdieu desarrolla en Meditaciones Pascalianas y en Contrafuegos $I^{19}$. Allí nuestro autor, además de apuntar a la importancia del capital simbólico y la crueldad de su privación, señala también como es a partir de este mismo capital desde donde se han de comprender los riesgos sociales del neoliberalismo.

El mayor problema, a juicio de Bourdieu, es precisamente que el neoliberalismo está legitimado socialmente gracias a su íntima relación con el cientifismo reinante. Los agentes neoliberales no solo son tolerados sino que gozan de legitimidad social, lo que les proporciona un fuerte poder de influencia.

Aquí es donde cobra especial actualidad el análisis de Bourdieu. En una situación donde el Estado es cada vez más débil, estos agentes neoliberales socialmente legitimados están en condiciones de ocupar su lugar en diversos aspectos y desde ahí, ya no solo influir a favor de sus intereses, sino configurar el medio social -y por ende los "habitus" de los ciudadanos con relación a ellos-. Pensemos, sin ir más lejos, en las principales empresas tecnológicas; que controlan y determinan los cauces por medio de los cuales los individuos llevan a cabo buena parte de sus tareas cotidianas y, además, almacenan la información fruto de esas tareas.

El sociólogo francés hablaba del Estado como legitimador último, pero cuando éste va siendo desplazado de esa función y las "teodiceas personales" —expresión

\footnotetext{
18 Weber, Max, Ética protestante y espíritu del capitalismo, México: FCE, 2011, pp. 247-248.

19 Bourdieu, Pierre, Contrafuegos I, Barcelona: Anagrama, 1999.
} 
bourdiana que aparece en Meditaciones Pascalianas - son provistas por entidades ajenas a él cabe poner especial atención a los intereses que pueda haber detrás de ellas y a la posible denigración de las condiciones sociales que se siga de su actuación. Bourdieu, en el citado libro, señala como el papel que las condiciones económicas - principalmente en su relación con el empleo- y como la privación de los ciudadanos de esas condiciones mínimas necesarias y de la posesión de un puesto de trabajo supone prácticamente la anulación social y existencial del mismo. Sin lugar a dudas, estas observaciones del teórico social son importantes, pero creo que su utillaje conceptual puede todavía llevarse más allá y ser especialmente útil para analizar aquellas situaciones no tan extremas en las que el individuo no se ve despojado de su papel en el juego, pero sí fuertemente determinado por la creciente influencia de poderosos agentes dentro del mismo que pudieran transformarlo para su interés.

Me refiero a la precarización general de las condiciones de vida que ocasiona el neoliberalismo y a su aceptación y asunción por parte de la población. Y es que, el riesgo principal del neoliberalismo no es solo la precarización sino su normalización y camuflaje. Este binomio precarización-normalización ha sido constante en las últimas décadas, pero puede alcanzar sus cotas más significativas con la inminente revolución tecnológica que muchos nos anuncian. Un ejemplo podemos encontrarlo en la amplia gama de plataformas virtuales que dan trabajo ya a un buen número de ciudadanos. Por un lado, ofrecen algo que escasea en la sociedad y, por el otro, lo hacen desde una perspectiva "buenista" y liberadora de las cargas tradicionales del trabajo - permiten realizar las tareas como dónde se quiera, garantizan ingresos inmediatos, liberan de horarios y jefes- Sin embargo, en el fondo se oculta la desaparición de buena parte de las garantías laborales que habían venido rigiendo las relaciones trabajador-empresario en los últimos años. El individuo -que hoy parece reclamar trabajos más flexibles y conciliables — es consciente de la precarización a la que es sometido, pero la mezcla entre el poder fáctico de esas empresas que ofrecen un trabajo necesario y la edulcoración de la precarización tienden a generar una aceptación conformista muy peligrosa y que gracias a Bourdieu podemos identificar con claridad ${ }^{20}$.

Con todo esto, volvemos a la indeterminación a la que el pensamiento de Bourdieu nos enfrentaba de cara al futuro. En este momento disruptivo que se nos anuncia, la acción y la orientación que el hombre haga de los elementos tecnológicos resultarán fundamentales, sobre todo de cara a la no incorporación de nuevas formas de dominación.

${ }^{20}$ Morozov, Evgeny, Capitalismo big tech... op. Cit. 


\subsection{Temporalización, sentido práctico e hipotéticos horizontes disruptivos}

EN ESTRECHA RELACIÓN con lo que venimos tratando, conviene volver a traer a colación el concepto bourdiano de temporalización y su íntima relación con la praxis. Quizá uno de los horizontes más prometedores de la nueva revolución tecnológica radique en la progresiva desaparición de aquellos trabajos más mecánicos y menos relacionados con lo típicamente humano como la creatividad, la empatía, las emociones ${ }^{21}$. Esto, que de primeras puede parecer aterrador dadas las perspectivas de paro que generaría, puede ayudar a afrontar el replanteamiento de la noción de trabajo que desde la época de Marx viene estando sobre la mesa. Para Bourdieu ${ }^{22}$, el concepto de trabajo como tarea subordinada a la obtención de un sustento es algo absolutamente relativo a un tipo concreto de mentalidad, la del homo oeconomicus, pero que para nada agota el sentido original y primigenio del trabajo. Más bien, lo difumina.

Su visión del trabajo va más allá, y entronca de pleno con los conceptos de tiempo y de práctica. A su vez, éstos dos van de la mano en el sentido de que la práctica hace al tiempo siempre y cuando se dé una correlación/complicidad ontológica entre el "habitus" y el medio social.

Sin embargo, esa complicidad ontológica puede romperse o dejar de darse. Esto ocurre cuando las expectativas dejan de corresponderse con la lógica del juego en el que se está inserto. Se vive entonces una situación de desfase en la que el individuo toma realmente conciencia del tiempo y lo hace mediante sensaciones como la impaciencia, la nostalgia, el descontento. Al desaparecer la ocupación que hace el presente, se rompe-siguiendo a Bourdieu- la correlación entre pasado y futuro $y$, por consiguiente, el individuo pierde toda noción de sentido/orientación vital.

A primera vista, la desorientación/ruptura con el juego podría suponer una oportunidad para la transformación del mismo gracias a la toma de conciencia. No obstante, Bourdieu nos señala que la toma de conciencia no es suficiente para ello, y que toda transformación requiere de la inserción en el juego mismo... "la demanda efectiva, requiere del poder efectivo" En este sentido, su pensamiento se vuelve iluminador a la hora de repensar las posibles oportunidades de disrupción que muchos filo-tecnología ven dibujadas en el futuro.

Fijémonos en las tesis de J. Rifkin del surgimiento de una nueva forma de organización económica social horizontal, y con ello de distintas formas de entender el trabajo más centradas en el desarrollo humano y personal ${ }^{23}$. Obviamente, siguien-

\footnotetext{
${ }^{21}$ Hidalgo, Manuel, El empleo del futuro, Barcelona: Deusto, 2018.

22 Bourdieu, Pierre, Estructuras sociales de la economía, Barcelona: Anagrama, 2018, pp. 13-27.

23 Rifkin, La sociedad de coste marginal 0 , op. cit.
} 
do los razonamientos de Bourdieu -y todo lo desarrollado arriba— éstas pecan de ingenuidad. La ruptura de la comunión ontológica habitus-realidad social como mucho puede generar grandes masas de "subproletarios" y movimientos milenaristas y populistas, pero difícilmente puede dar lugar a nuevas formas de organización y gestión socio-económica. Un futuro más esperanzador en ese sentido únicamente puede ir de la mano de la progresiva transformación de los "habitus" dentro del mismo juego, por medio de una educación orientada intencionadamente en esa dirección. La transformación social solo puede ser efectiva mientras que no se rompa la temporalización, esto es, mientras exista cierta complicidad ontológica entre "habitus" y medio social; por ello, los horizontes disruptivos pueden convertirse en quimeras si solamente remiten al futuro y dejan de lado la labor que debe hacerse en el presente.

\subsection{Capital económico, capital cultural y mantenimiento del statu quo}

PARA TERMinar, EL CONCEPTO DE BOURDiAno de capital todavía nos permite darle una vuelta de tuerca más a nuestro análisis. Teniendo en cuenta la estrecha relación que existe para el francés entre la posición en la distribución de los capitales y la temporalización, es necesario reflexionar acerca de la distribución de éstos de cara al futuro inmediato así como de las posibles formas de transmisión que pudieran darse de cara a una hipotética "Revolución tecnológica".

Los principales discursos filo-tecnológicos hablan del potencial de Internet y de las energías renovables para romper con el capitalismo tal como lo conocemos gracias a la irrupción del coste marginal 0 , o sea, el intercambio gratuito de productos y servicios que terminaría con la productividad que hace viable el mercado capitalista tradicional. En este sentido, se profetiza un sistema de organización horizontal en el que ninguna organización/empresa congregaría grandes cantidades de capital, sino que los mismos ciudadanos conectados a la red producirían e intercambiarían desde elementos culturales hasta la misma energía eléctrica.

El pensamiento del sociólogo francés nos conduce, de nuevo, a una serie de objeciones con relación a esta cuasi utópica visión de futuro: 1) habría que tomar en consideración las formas en que los distintos tipos de capital se entrelazan. Esto es, como el capital económico se relaciona con el capital cultural -tanto incorporado como objetivado- y cómo éstos lo hacen a su vez con el capital social. 2) habría que considerar los mecanismos de transmisión del capital que puedan estar en funcionamiento. 
Pensar en una sociedad donde el capital se distribuya horizontalmente entre los ciudadanos supone un cambio total -utópico- con respecto al actual estado de cosas. Implica pensar que las grandes empresas trasnacionales hayan desaparecido y cedido su poder y capital sin ningún tipo de resistencia. Siguiendo los planteamientos de Bourdieu, esto no sería realista en absoluto ya que, a su juicio, "el medio social posee una especie de conatus que tiende a reproducir las estructuras de la distribución del capital y las posibilidades de beneficio correlativo" ${ }^{24}$. Este conatus actúa a partir del entrelazamiento existente entre los distintos tipos de capital y la consiguiente transmutabilidad de todos ellos con el económico.

De cara a nuestra preocupación, resulta de especial interés el fijarse en el capital cultural. Hablamos de una revolución de las tecnologías dónde éstas jugarán un papel cada vez mayor en todos los ámbitos; ahora bien, es importante atender a una doble cuestión: la propiedad de esos medios tecnológicos y la habilidad para usarlos. Según el sociólogo francés, el capital cultural converge con el económico en el sentido de que se precisan recursos económicos para poder dedicar el tiempo necesario para obtener capital cultural. Pero no sólo eso, también hacen falta recursos económicos para hacerse con los diferentes medios materiales que soportan el capital cultural.

Por tanto, si con Bourdieu hablamos de la existencia de cierto conatus en el medio social, seríamos muy ingenuos si no cayésemos en la cuenta de las formas de transmisión del capital que se puede producir de cara al futuro inmediato. Lógicamente, el capital económico que ahora está concentrado verticalmente en grandes empresas trasnacionales, puede seguir estándolo en el futuro en empresas similares que hayan aprovechado su posición para acumular capital cultural en forma de medios tecnológicos --datos, software de todo tipo, contadores inteligentes, etc.- . De hecho, sería más ingenuo aun considerar que los actuales poseedores del capital no hagan movimientos en esa dirección.

\section{Conclusiones}

El objetivo principal de este artículo ha sido señalar la utilidad de la teoría social de Pierre Bourdieu. Más allá del debate "pro-" "contra-" se ha pretendido poner sobre la mesa una serie de herramientas teóricas desde las que analizar en profundidad las implicaciones sociales de las nuevas tecnologías. En este sentido se concluye lo siguiente:

24 Bourdieu, Pierre, Meditaciones Pascalianas, op. cit. p. 285. 
1) La perspectiva teórica bourdiana se vuelve especialmente fecunda a la hora estudiar las distintas formas en que la dominación social puede tener lugar. De este modo, constituye una útil herramienta para profundizar en la relación tecnología-dominación, cuyas implicaciones van mucho más allá de la mera sustitución hombre/máquina.

2) Tres conceptos resultan de especial utilidad a la hora de analizar las implicaciones sociales de la hipotética revolución tecnológica: 1) el "habitus"; 2) el tiempo y 3) el capital. El concepto de "habitus" permite indagar en la estrecha relación existente entre la estructura del medio social y la propia corporalidad individual. Las disposiciones inconscientes del individuo, su propio cuerpo, son moldeados por el medio social en el que está inmerso y, por consiguiente, por las fuerzas y poderes dominantes en él. Esto se lleva a cabo en todas las actividades de la vida cotidiana, hasta el punto de que los limites y posibilidades del mundo social coinciden con los limites $y$ expectativas individuales. En esta dirección, cabe prestar especial atención al potencial que las empresas trasnacionales y las propias empresas tecnológicas pueden para determinar el medio social y, con ello, la propia interioridad de los individuos. Poniendo especial éénfasis en la necesidad de instancias educativas que se adelanten en esa labor contrarrestando el poder de estos agentes y llevando a cabo procesos educativos empoderadores de los ciudadanos.

La concepción bourdiana del tiempo puede sernos especialmente útil a la hora de comprender la profunda dimensiónn antropológica y existencial de la práctica y, por ende, del trabajo. De esta manera, puede ayudarnos a prevenir aquellas presiones sociales conducentes a la precarización; y a tomar conciencia de que la única vía posible de cambio no radica en la ruptura -que supondría la paralización del tiempo- sino en la progresiva transformación desde dentro. La Revoluciónn tecnológica que se presenta como una oportunidad para el cambio sólo puede ser tal si existe una transformación progresiva de las mentes y los cuerpos, dentro del mismo tiempo -o sea dentro del mismo juego-.

La íntima relación entre capital e historia y las distintas formas de capital transmutables entre sí- adquiere gran utilidad a la hora de reflexionar acerca de las formas de transmisión del capital y cómo éstas pueden operar en una época de cambio tecnológico. Las utopías de un "futuro horizontal" pueden darse de bruces con una verticalidad aun más peligrosa por medio de la transmutación capital económico-cultural-económico. 
Con todo esto, se aprecia que el pensamiento de Bourdieu pone sobre la mesa un reto principal de cara al futuro: el de prestar especial atención a las formas en que los distintos agentes neoliberales intentan universalizar su interés grupal -las tecnologías pueden muy eficaces en ese sentido-. La debilidad del Estado como legitimador de puntos de vista (geómetra de todas las perspectivas) abre la puerta a que otras instancias puedan ocupar ese espacio o valerse del mismo Estado para obtener sus fines. La teoría social ayuda a tomar conciencia de ello, ahora bien, en la práctica son necesarias iniciativas e instituciones que contrarresten a estos agentes, que cuentan ya con importante legitimidad social. 


\section{REFERENCIAS BIBLIOGRÁFICAS}

Bologna, S. The rise of the self-employed workforce. Mímesis. 2018.

Bourdieu, P. Contrafuegos I. Barcerlona: Anagrama.1999.

Bourdieu, P. El sentido práctico. Madrid: Siglo XXI. 2009.

Bourdieu, P. Espacio social y poder simbólico. En P. Bourdieu, Cosas dichas (pp. 127-142). Barcelona: Gedisa. 1988.

Bourdieu, P. Las estructuras sociales de la economía. Barcelona: Anagrama. 2000.

Bourdieu, P. Meditaciones Pascalianas. Barcelona: Anagrama. 1999.

Bourdieu, P. Poder, derecho y clases sociales. Bilbao: Desclée de Brouwer. 2001.

Bourdieu, P. Razones prácticas. Barcelona: Anagrama. 1997.

Fernández, J. M. "Capital simbólico, dominación y legitimidad. Las raíces weberianas de la sociología de Pierre Bourdieu”. Papers, revista de sociología, vol. 68, no 1, 2015, pp. 33-60.

George, S. El Informe Lugano II. Barcelona: Deusto. 2013.

George, S. Los usurpadores. Barcelona: Deusto. 2014.

Hidalgo, M. El trabajo del futuro. Barcelona: Deusto. 2018.

Mauss, M. Concepto de técnica corporal. En Sociología y antropología. Madrid: Tecnos. 1979.

Morozov, E. Capitalismo big tech, ¿welfare o neofeudalismo digital? Madrid: Enclave de libros. 2018.

Rifkin, J. El green new deal global. Barcelona: Paidós. 2018.

Rifkin, J. La sociedad de coste marginal 0. Barcelona: Paidós. 2014.

Schwab, K. La cuarta revolución industrial. Madrid: Debate. 2016.

Weber, M. La ética protestante y el espiritu del capitalismo. Mexico: FCE. 2011.

DOI: https://doi.org/10.15366/bp2021.27.014

Bajo Palabra. II Época. № 27. Pgs: 273-290 